\title{
Philosophiques
}

\section{Sur le développement du concept de l'intentionnalité chez Brentano et Husserl}

\section{Peter McCormick}

Volume 8, numéro 2, octobre 1981

URI : https://id.erudit.org/iderudit/203167ar

DOI : https://doi.org/10.7202/203167ar

Aller au sommaire du numéro

Éditeur(s)

Société de philosophie du Québec

ISSN

0316-2923 (imprimé)

1492-1391 (numérique)

Découvrir la revue

Citer cet article

McCormick, P. (1981). Sur le développement du concept de l'intentionnalité chez Brentano et Husserl. Philosophiques, 8(2), 227-237.

https://doi.org/10.7202/203167ar
Résumé de l'article

La théorie de l'intentionnalité de Brentano a des sources thomistes et cette provenance lui confère un penchant réaliste, en ce sens qu'elle n'exclut aucunement une relation entre les objets intentionnels et les objets physiques existant dans le monde. Par contre, la façon dont Husserl a interprété la théorie de Brentano révèle un penchant vers l'idéalisme puisque, d'après son explication, il ne peut y avoir aucune relation nécessaire entre cette catégorie d'objets intentionnels, que nous appelons des perceptions, et les objets physiques existant dans le monde. 


\title{
SUR LE DÉVELOPPEMENT DU CONCEPT DE L'INTENTIONNALITÉ CHEZ BRENTANO ET HUSSERL*
}

\author{
par Peter McCormick
}

\begin{abstract}
RÉSUMÉ. La théorie de l'intentionnalité de Brentano a des sources thomistes et cette provenance lui confere un penchant réaliste, en ce sens qu'elle n'exclut aucunement une relation entre les objets intentionnels et les objets physiques existant dans le monde. Par contre, la façon dont Husserl a interprété la théorie de Brentano révèle un penchant vers l'idéalisme puisque, d'après son explication, il ne peut $y$ avoir aucune relation nécessaire entre cette catégorie d'objets intentionnels, que nous appelons des perceptions, et les objets physiques existant dans le monde.
\end{abstract}

\begin{abstract}
Brentano's theory of intentionality is of scholastic origin, which confers on the theory a "realist" tendency, in the sense that the theory does not exclude relations between intentional objects and physical objects. By contrast, Husserl's way of interpreting Brentano shows an idealist strain, but in the sense that there can be no necessary relations between perceptions and physical objects. The major difficulty is that the theory is too general and the central concept too ambiguous.
\end{abstract}

Le concept d'intentionnalité, qui est la clé de la distinction du psychique et du physique chez Brentano, doit être compris d'après sa source et en fonction de son influence. Cette source est scolastique, notamment thomiste, et cette influence phénoménologique, notamment husserlienne. Pour évaluer de façon critique la convenance de ce concept d'intentionnalité, nous le situerons par rapport à cette double perspective. Notre stratégie consiste à relever les éventuelles faiblesses critiques de l'intentionnalité par l'examen minutieux de son origine et de l'accueil qui lui a été réservé.

* Version révisée d'un texte présenté au Congrès de l'ACFAS, à Québec, mai 1980, dans le cadre d'une table ronde sur L'intentionnalité: phénoménologie vs positivisme et philosophe analytique. 


\section{BRENTANO}

C'est en grande partie dans l'histoire que Brentano a puisé la notion d'intentionnel ${ }^{1}$. Il le reconnaît pleinement lui-même dans les longues notes qui sont presque toujours omises lorsqu'on cite ses explications sur l'intentionnalité et qui figurent dans son livre Psychologie du point de vue empirique $(1874)^{2}$. L'influence prépondérante a bien sûr été celle de Thomas d'Aquin ${ }^{3}$. Il est évident, en effet, que c'est chez l'Aquinate que Brentano a puisé la première des deux notions qui forment son concept d'intentionnalité, la notion d'«inexistentia intentionalis». Les auteurs ne s'entendent pas sur la provenance du deuxième élément constitutif de ce concept, la notion de «référence à un contenu, visée d'objet ... objectivité immanente." Spiegelberg a soutenu, dans sa History of the Phenomenological Movement, que la notion de référence à un objet était un apport original de Brentano. Dans le sens contraire, A. Marras a prouvé de façon convaincante que cette notion n'est pas originale puisque «... non seulement elle n'est pas incompatible avec l'idée scolastique de l' «inexistentia intentionalis», mais elle est en fait constitutive de cette idée ${ }^{4} »$. Sans entrer dans le détail de cette controverse, je crois que, sur la foi des arguments présentés jusqu'ici, nous devons conclure provisoirement à l'origine scolastique des deux éléments principaux de la théorie de Brentano; ils ne proviennent pas de Brentano luimiême.

Cette discussion sur la provenance de la doctrine de Brentano n'est pas étrangère à notre préoccupation immédiate d'une évaluation critique du concept de l'intentionnalité. Elle attire en effet notre attention sur l'une des plus importantes questions que nous pouvons soulever concernant la convenance du concept

1. Sur la doctrine de Brentano voir notamment R.M. Chisholm, «Brentano's Descriptive Psychology and the Intentional», dans Phenomenology and Existentialism, éd. M.H. Mandelbaum et E.N. Lee (Baltimore, 1967); R.M. Chisholm et W. Sellars, «Intentionality and the Mental", dans H. Feigl, et al. éds, Concepts, Theories, and the Mind-Body Problem (Minneapolis, 1958); L. MCAlister, "Franz Brentano and Intentional Inexistence", Journal of the History of Philosophy, 8(1970), 423-430; et J.C. Morrison, "Husserl and Brentano on Intentionality", Philosophy and Phenomenological Research, 31 (1970), 27-46. Les deux lectures de Chisholm sont défectueuses.

2. Voir la deuxième édition allemande, Psychologie vom empirischen Standpunkt, Leipzig 1924 et la traduction française de M. De Gandillac, Psycbologie du point de vue empirique, Paris, Aubier, 1944

3. Voir A. Marras, "Scholastic Roots of Brentano's Conception of Intentionality", dans L. McAlister, éd., The Pbilosophy of Brentano (Londres, 1976), pp. 123-139.

4. Marras, op, cit., p. 129. 
d'intentionnalité de Brentano. Et cette question, comme le montre très clairement Marras, est de savoir si la notion d' «inexistentia intentionalis» entraîne la dépendance existentielle de tout objet connu. Bref, le problème est de savoir si cette notion présuppose une épistémologie idéaliste plutôt que réaliste. Dans l'affirmative, comme le pense Spiegelberg, la reconstruction du concept de l'intentionnalité ne peut tirer profit de toutes les possibilités qu'offre ce concept $t^{5}$, car l'existence indépendante de l'artefact en serait fatalement compromise. Après avoir analysé à fond les textes scolastiques auxquels Brentano renvoie, Marras conclut que Spiegelberg s'est trompé à ce sujet. On peut en effet tenir la doctrine de l' "inexistentia intentionalis» et donner une explication connexe de l'existence indépendante des objets non mentaux; car cette doctrine ne nous engage qu'à poser l'existence d'une représentation (species ou forme) de l'objet. Et cette représentation, "la species ou l'intentio, n'est pas ce qui (id quod) est directement ou d'abord connu par l'entendement (comme l'est 'l'expression' ou 'l'idée' de l'empirisme classique), mais est plutôt ce par quoi (id quo) l'objet extramental est connu. C'est-à-dire que la species est le véhicule qui porte la référence à l'objet extramental non immanent, qui seul est fondamentalement connu ${ }^{6}{ }$. Le texte essentiel sur lequel s'appuie cette interprétation convaincante de l' «inexistentia intentionalis» est celui de Thomas d'Aquin: «Species intelligibilis, écrit ce dernier, non est quod intelligitur sed id quo intelligit intellectus ${ }^{7} »$. (Voir dans un autre sens les passages, par exemple $S T, I, 81,1$, où Thomas d'Aquin écrit: «... Actus enim apprehensivae virtutis non ita proprie dicitur motus, sicut actio appetitus: nam operatio autem virtutis apprehensivae perficitur in hoc, quod res apprehensivae sunt in apprehendente...»).

Quels que soient donc les termes réels utilisés par Brentano dans ses écrits philosophiques tardifs (la prétendue Immanenzkrisis

5. Voir H. Spiegelberg, “'Intention' and 'Intentionality' in the Scholastics, Brentano, and Husserl", dans McAlister, éd. (1976), Pp. 108-127, et The Phenomenological Movement, $3^{\mathrm{e}}$ édition, (La Haye, 1975).

6. Matras, op. cit., p. 138.

7. Somme Théologique, I, 85, 2, citée dans Marras, p. 138, note 34. Un lecteur anonyme suggère que "l'étude de la doctrine de F. von Brentano pourrait être approfondie, en particulier en ce qui concerne la distinction entre les phénomènes physiques et psychiques (distinction que Husserl conteste sur plusieurs points, notamment à propos des caractéristiques des phénomènes psychiques, de l' «inexistentia intentionalis" et du rapport à un objet). Pour cette étude, les suppléments rendus publics par O. Kraus en 1924 sont fort utiles.» 
de 1905 au cours de laquelle Brentano a cessé de croire à des objets dépendants-de-la-pensée, c'est-à-dire à des entités idéales qui auraient été les seuls référents des actes mentaux, au profit de sa prétendue affirmation "réistique» d'objets indépendantsde-l'esprit, c'est-à-dire d'entités réelles qui seraient ses référents), il est évident qu'il n'y a aucune incompatibilité à soutenir que son concept d'intentionnalité, tel que nous nous en sommes servi, nous permet de tenir en même temps à l'existence d'entités réelles, référents des actes mentaux.

S'il y a une lacune fondamentale dans la notion d'intentionnalité, elle doit être cherchée ailleurs que dans son origine, en fin de compte scolastique.

\section{DE BRENTANO À HUSSERL}

Se pourrait-il que la transmission de ce concept de Brentano à Husserl nous instruise ${ }^{8}$ ?

Le principal texte est ici la discussion de la doctrine de Brentano au début du chapitre II de la $\mathrm{V}^{\mathrm{e}}$ Recherche logique de Husserl $1^{9}$. Nous devons donc prêter attention à ce qui est effectivement dit ici, comme nous l'avons fait dans l'interprétation de Brentano et des scolastiques.

Mais, il est difficile de savoir ce qui est dit, pour une double raison. Tout d'abord parce que Spiegelberg a écrit sur ce sujet, il y a plus de quarante ans, une étude très influente intitulée: "Der Begriff der Intentionalität in der Scholastik bei Brentano und bei Husserl». Publié dans les Philosopbische Hefte, V (1936), ce texte a été réimprimé en allemand dans les Studia Pbilosophica, XXIX (1970) et traduit en anglais en 1976 avec très peu de modifications ${ }^{10}$. Malgré le poids de cet article, nous ne pouvons, sans examen personnel du texte de Husserl, accepter d'emblée les idées de Spiegelberg. Car nous venons en effet de voir qu'il s'était trompé sur l'origine et le sens de la doctrine de l'«inexistentia intentionalis» dans l'histoire du mouvement phénoménologique.

8. En général, voir W. Del Negro, «Von Brentano über Husserl zu Heidegger», Zeitchrift für philos. Forschung, 7(1953) et A. Kastil, «Brentanos Stellung zur Phänomenologie», Zeitschrift fïr philos. Forschung, 5 (1951).

9. Traduit en anglais par J. Findlay (New York, 1970).

10. Voir note 5 . 
Deuxièmement, un retour aux textes ne va pas sans ses problèmes propres. La $\mathrm{V}^{\mathrm{e}}$ Recherche logique de Husserl a connu six éditions, depuis la première en 1901 jusqu'à la sixième en 1975 qui restaurait le texte original de 1901 , en passant par les éditions revues de 1913 et les réimpressions sans révisions en 1922, 1928 et 1968, de l'édition de 1913. Mais une septième édition est en préparation et fait partie de l'édition critique des Recherches logiques dans la série Husserliana. Ce n'est pas tout. L'étude des observations de Husserl sur Brentano dans cette $\mathrm{V}^{\mathrm{e}}$ Recherche logique ne peut se faire dans l'oubli d'une autre série d'observations qu'il a faites sur des thèmes analogues dans le texte Aüßere und innere W abrnebmung: physische und psychische Pbänomene et qu'il a ajouté en appendice à la VI ${ }^{\mathrm{e}}$ Recherche logique, article encore plus compliqué sur le plan textuel. Grâce aux recherches effectuées jusqu'ici à ce sujet, je crois cependant que nous n'avons à retenir que les versions de 1901 et de 1913 . L'appendice de la $\mathrm{VI}^{\mathrm{e}}$ Recherche traite pratiquement de toutes les questions abordées dans ces versions et je crois donc que nous sommes justifiées à le rejeter.

\section{HUSSERL SUR L'INTENTIONNALITÉ}

Quel est donc le fond de la pensée de Husserl sur l'intentionnalité de Brentano?

D'après Spiegelberg, cette pensée se ramène à une double mise en garde contre deux mauvaises interprétations possibles de la doctrine de Brentano et à quelques précisions de Husserl sur sa propre doctrine. Spiegelberg estime que Husserl a emprunté à Brentano l'idée d'intentionnalité entendue comme rapport à un objet, sans la notion d' «inexistentia intentionalis». Il ne faut cependant pas comprendre ce rapport, nous prévient immédiatement Husserl, comme un rapport existant entre des expériences réelles qui présupposeraient à leur tour «l'existence d'une relation réelle entre le moi et l'objet connu ${ }^{11}$ ». Une telle relation réelle lui aurait été inacceptable au cours des deux années après la découverte de 1905 . Husserl nous avertit, en second lieu, qu'il ne faut pas non plus concevoir l'objet de ce rapport ou de cette orientation de façon «immanente», à la manière dont les données de l'essence et les apparences sont dites immanentes, mais bien de façon "transcendante et en un sens qui reste à montrer ${ }^{12}$ ».

11. Spiegelberg, op. cit., p. 123.

12. Spiegelberg, op. cit., p. 123. 
Outre ces précautions, Spiegelberg explique que Husserl insiste sur «les vécus intentionnels» plutôt que sur les «objets intentionnels», c'est-à-dire «certaines catégories d'objets se rapportant aux vécus» (Husserl, $V^{\mathrm{e}}$ Recherche logique, section 13); qu'il insiste plus que Brentano sur le fait que les expressions linguistiques sont des porteurs d'intentionnalité et non de simples actes mentaux; qu'il donne au terme «intention» appliqué aux objets un sens plus précis que Brentano, «l'intentionnel» (par opposition à reel, que renferme le vécu) renvoyant à «tout ce qui est situé au-delà du monde des vécus et de leurs 'composantes' mais qui est encore vraiment visé ou dans l'intention des actes comme 'corrélats' ${ }^{\prime 3}{ }_{»}$; et enfin que Husserl donne de l'intentionnalité une interprétation presque idéaliste lorsqu'il s'en sert relativement à des objets pour désigner «le mode d'être de ces objets, dont l'existence dépend de la conscience, par opposition au mode d'être de la réalité" (Spiegelberg, 124), explication qui a conduit Husserl à confondre intentionnalité comme visée d'un objet et intentionnalité comme constitution de l'objet.

Bref, Spiegelberg estime que Husserl corrige la notion d'intentionnalité de Brentano en insistant sur le caractère constituant de l'acte d'intentionnalité et sur la dimension immanente de l'objet d'intentionnalité. Si tel est vraiment le fond de la pensée de Husserl sur Brentano, nous devons conclure, je crois, qu'il s'est trompé dans ses corrections. Insister sur le caractère non immanent de l'objet de l'intentionnalité, c'est simplement se méprendre sur la doctrine scolastique de l'intentio chez Brentano pour qui, comme nous le rappelle Marras, elle est «un «ens rationis», un universel et par conséquent un attribut . . (ayant), à la différence des particuliers, un mode d'existence dépendant ${ }^{14}{ }^{\prime}$, ce qui n'empêche pas son contenu d'être relié à des objets existant indépendamment. Et tenir au caractère constituant de l'intentionnalité est une méprise de Husserl et ne représente aucunement la pensée de Brentano.

Une analyse plus serrée des textes de Husserl nous fournirat-elle d'autres éléments que ceux retenus par Spiegelberg?

Tout d'abord, laquelle des deux éditions peut être considérée comme normative en la matière? Si nous comparons les

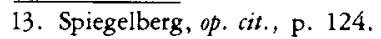

14. Marras, op. cit., p. 139. 
principaux passages où Husserl analyse les doctrines de Brentano, nous y relevons trois différences, dont l'une est relativement peu importante puisqu'il s'agit d'une note ajoutée par Husserl à sa discussion du «meinen» au chapitre II, paragraphe II des Recherches logiques. Une deuxième modification concerne le titre du chapitre II; le titre original «Bewußtsein als psycbiscber $A k t$ " de l'édition de 1901 devient "Bewußtsein als intentionales Erlebnis» dans l'édition de 1913. Cette modification est bien conforme à la discussion husserlienne de l'intentionnalité, puisque, comme l'a remarqué Spiegelberg, Husserl insiste davantage sur les vécus que sur les actes. Mais, comme c'est précisément au moment de la parution de la deuxième édition que la pensée de Husserl a subi l'attrait de l'idéalisme (voir notamment Ideen I et le projet d'une Préface aux Recherches logiques de 1913), nous devons souligner toute l'importance du premier titre dans le cadre de sa discussion de Brentano. Ainsi, l'intentionnalité chez Brentano est perçue comme une réalité d'ordre psychologique.

Mais la dernière modification est la plus importante. Elle apparaît au paragraphe 10 , là où Husserl a ajouté une très longue phrase et une longue note explicative sur le sens de ses observations visant la définition des "phénomènes psychiques» de Brentano. Husserl rejettera cette phrase au paragraphe II, chacun de ses termes lui paraissant sérieusement trompeur. Ses observations sont teintées d'idéalisme lorsqu'elles font appel à des concepts comme ceux de «rein phänomenologische Gattungsidee, " «intentionales Erlebnis», «Apperzeption» et «reine Begriffe der Erlebnisse ${ }^{15}$ ». C'est pourquoi il est plus indiqué, me semble-t-il, de s'en tenir à l'énoncé de la conception husserlienne de la première édition de 1901. J'en conclus donc qu'il n'est pas déraisonnable de ne retenir comme normatif, pour la lecture husserlienne de l'intentionnalité chez Brentano, que le texte de la première édition.

L'explication husserlienne comporte-t-elle des éléments que nous pourrions utiliser pour notre analyse critique de l'idée de l'intentionnalité?

Au paragraphe 9 de son chapitre sur «la conscience en tant que vécu intentionnel, Husserl fait l'éloge de la Psycbologie de Brentano, dont l'importance est fondamentale. Il aborde ensuite,

15. Logische Untersuchugen (1913), p. 369 
au paragraphe 10, « la caractérisation descriptive des actes en tant que vécus 'intentionnels'». Il faut comprendre que Husserl utilise ici le terme «psychique» comme l'équivalent de «mental». Le raisonnement est donc le suivant: l'essence de la conscience c'est le psychique, et l'essence du psychique, l'intentionnalité. Après avoir discuté des seules caractéristiques intentionnelles du psychique, Husserl cite longuement la Psychologie de Brentano (sans les notes où figuraient les importants renvois scolastiques). Et c'est la notion de Weise (mode) qui, dans ce texte de Brentano, suscite son commentaire sur le rapport de l'acte intentionnel à son objet.

Ce qui frappe Husserl, c'est que le discours de Brentano sur les modes donne à penser qu'il existe des espèces spécifiquement différentes de relations entre les actes intentionnels et leurs objets. Et ces différences relationnelles peuvent être définies autrement qu'en faisant simplement appel aux différences entre les objets intentionnels. Ainsi, ce qui est propre à cette relation elle-même dans des actes intentionnels aussi différents que ceux de représenter, de juger ou d'aimer (pour reprendre des exemples tirés de chacune des trois catégories fondamentales de Brentano que Husserl adopte sans autre), ce n'est pas nécessairement l'objet représenté, l'objet jugé ou l'objet aimé, mais le mode de structuration de la relation acte-objet. «Die Weise, écrit Husserl, in der eine 'blo $\beta$,e Vorstellung' eines Sachverhalts dessen ihren 'Gegenstand' meint, ist eine andere, als die Weise des Urteils, das den Sachverhalt für wahr oder falsch hält ${ }^{16}$ ». Et bien que de nombreux actes intentionnels soient complexes, c'est-à-dire comprennent plus qu'une des trois espèces fondamentales d'actes psychiques (Husserl prend l'exemple des actes psychiques émotifs d'aimer ou de haïr), cette complexité est néanmoins réductible, d'après Husserl, à «une caractéristique intentionnelle primitive» qui ne peut être proprement décrite par référence, au-delà d'ellemême, à d'autres expériences psychiques. L'unité de tels actes psychiques complexes provient de l'essence de leurs caractéristiques intentionnelles primitives. Et cette unité essentielle ellemême de l'une des trois espèces fondamentales particulières d'actes psychiques ne peut s'expliquer par référence à certaines caractéristiques des autres espèces.

16. Logische Untersucbugen (1901), p. 27. Ci-après: $L U$. 
Husserl propose ensuite un exemple important qu'il ne nous sera pas inutile d'avoir à portée de la main.

So ist z. B. die ästhetische Billigung oder Misbilligung eine Weise intentionaler Beiziehung, die sich als evident eigenartig erweist gegenüber dem bloß̉en Vorstellen oder theoretischen Beurteilen des ästhetische Objekts. Die ästhetische Billigung kann zwar ausgesagt werden, und die Aussage ist ein Urteil und schlie $\beta$ t solche Vorstellungen ein. Aber dann ist die ästhetische Intention, ebenso wie ihr Objekt, Gegenstand von Vorstellungen und Urteilen; sie selbst bleibt von diesen theoretischen Akten wesentlich verschieden ${ }^{17}$.

Bref, il y a au moins trois espèces différentes et fondamentales d'intentionnalité.

Dans la dernière section de son explication à ce sujet, Husserl dissocie la nature du contenu d'un objet intentionnel de celle du contenu d'un objet perceptuel. Seul le premier est objet $d^{\prime}$ intention comme un tout ${ }^{18}$. Il n'est absolument pas question, n'en déplaise à Spiegelberg, d'existence dépendante ou indépendante. Pour établir sa distinction, Husserl ne fait que renvoyer à la distinction plus générale des parties et des touts, et s'appuie implicitement sur l'examen détaillé qu'il en a donné dans la $\mathrm{III}^{\mathrm{e}}$ Recherche logique ${ }^{19}$.

\section{EN GUISE DE CONCLUSION}

$Y$ a-t-il un aspect de l'explication de Husserl qui, par opposition à la lecture très influente de Spiegelberg, aurait une signification pour notre recherche?

17. $L U$, p. 28 .

18. $L U$, p. 20 .

19. Sur divers problèmes techniques voir R.E. Aquila, «The Status of Intentional Objects», The New Scholasticsm, 45 (1971); R.B. Arnaud, «Brentanist Relations», dans K. Lehrer, éd., Analysis and Metaphysics (Dordrecht, 1975) et R. Kamitz, "Acts and Relations in Brentano", Analysis, 22 (1962). Signalons cependant les réflexions suivantes d'un lecteur anonyme: "En ce qui concerne la perception externe (äussere Wahrnehmung), certes, la «Erlebniz" dont parle Husserl est plus qu'une simple «inexistentia intentionalis", plus qu'un simple rapport à un objet de chez Brentano. Si Husserl met l'accent, du moins dans la Ve recherche, sur l'acte intentionel dans son rapport à un objet, il ne nie pas la réalité extra-mentale, puisqu'il parle de «an die Sache selbst» et fait la distinction entre objet intentionnel (intentional Objekt) et contenu intentionnel (intentional Inhalt). C'est un thème que Husserl reprendra lorsqu'il précisera les trois types d'intuition: sensible, éidétique et catégoriale. La lecture attentive des textes de Husserl de cette période (LU) ne nous autorise pas à affirmer que les objets extra-mentaux (wirkliche) ne sont que les corrélats des actes intentionnels, mais plutôt que l'accès à la réalité extra-mentale ou mieux encore l'apparition des choses extra-mentales se fait selon les perspectives ou les visées de la conscience. Voilà un nouveau sens du réalisme qui ne correspond ni à celui de Thomas d'Aquin ni à celui d'Aristote.» 
Quelle que soit l'orientation ultérieure de la pensée de Husserl au moment où il refondait ces passages en 1913, il est déjà évident dans ce texte de 1901 que, pour lui, la notion de visée d'un objet de Brentano implique une dépendance pas rapport à l'esprit. Le seul objet que présuppose la relation intentionnelle, que cette relation soit sous le mode représentatif, de jugement ou émotif, est un objet psychique. Il est vrai que celui-ci (comme dans le cas d'un objet perceptible, mais non de la représentation de Jupiter pour reprendre l'exemple de Husserl au paragraphe 11) peut en outre être relié à un objet dans le monde qui ne dépende pas de l'esprit. Mais cette seconde relation n'est que contingente. La doctrine de l'intentionnalité est par conséquent une doctrine psychologique en ce sens que quels que soient les objets en cause, ils sont ou bien nécessairement reliés comme des corrélats à l'existence d'actes psychiques seulement, ou ne sont reliés que de façon contingente à l'existence possible d'objets physiques dans le monde. Ici comme là, tout ce qui peut exister ou non dans le monde n'a aucun rapport nécessaire avec l'esprit. C'est peut-être justement cette faiblesse que Brentano a perçue relativement à sa propre doctrine de l'intentionnalité, qui l'a poussé, vers 1905, à réorienter son épistémologie dans le sens du réisme de Kotarbinski. On peut trouver un peu ironique que, peu de temps après, Husserl aille imprimer à sa propre épistémologie un sens opposé dans Ideen I (1913).

Résumons-nous. Nous avons vu que la théorie de l'intentionnalité de Brentano avait des sources thomistes et que cette provenance lui confere un penchant réaliste en ce sens qu'elle n'exclut aucunement une relation entre les objets intentionnels, quels qu'ils soient, et les objets physiques existant dans le monde. Par contre, la façon dont Husserl a interprété la théorie de Brentano révèle un penchant vers l'idéalisme puisque, d'après son explication, il ne peut y avoir aucune relation nécessaire même entre cette catégorie d'objets intentionnels, que nous appelons des perceptions, et les objets physiques existant dans le monde.

La principale difficulté provient une nouvelle fois de la trop grande généralité de la théorie en question. Brentano lui-même, en effet, ne sait pas de façon absolument certaine quels sont les objets pouvant se rapporter aux actes intentionnels ni quelles relations ces objets peuvent avoir, non pas avec la conscience, 
mais avec le monde matériel: ainsi, sa théorie de l'intentionnalité demeure trop vague. En d'autres termes, les deux notions constitutives de la théorie de l'intentionnalité chez Brentano, l' «inexistentia intentionalis» et la visée d'un objet, ne sont pas analysées à fond, puisque ni leur nature ni les rapports spécifiques qu'ils peuvent entretenir ne sont clairement explicités.

Les différentes perspectives que cet examen de l'origine et de la réception de la théorie de Brentano a ouvertes, s'expliquent assez simplement par le fait que le concept cardinal de Brentano est ambigu. Il s'ensuit que la version revue de cette théorie chez Husserl, malgré ses propres difficultés, doit également l'être. Mais une conclusion encore plus importante peut également être dégagée: le caractère ambigu de la théorie de Brentano voile un autre de ses aspects fondamentaux. Et dans la mesure où ce caractère ambigu lui est inhérent, nous sommes en fait en présence d'un psychologisme. Cette ambiguïté provient justement de la notion de référence à un objet non spécifié, notion qui, à son tour, présuppose la seule autre notion du concept, à savoir l'«inexistentia intentionalis». Pour lever l'ambiguïté du concept d'intentionnalité chez Brentano, il faudrait donc se débarrasser de la notion trop générale de référence à un objet, mais, ce faisant, on détruirait le concept lui-même. Ainsi, le concept de Brentano est d'une ambiguïté inexorable. Pourrait-il être récupéré à l'aide d'un autre discours, d'un discours qui n'est plus phénoménologique ${ }^{20}$

Département de philosophie

Faculté des Arts

Université d'Ottawa

20. Je tiens à remercier les lecteurs anonymes pour les observations critiques qu'ils m'ont faites sur une version antérieure du présent article. 